zeichniss , mit Berichtigung ., des früheren von. Cabanis publicirten, an denselben zur Veröffentlichung senden. Dieses Verzeichniss soll dann mit Noten über. Vorkommen, ob Zugvogel, Strichvogel oder Standyogel u. sti w. etwa so wie in der $\mathbf{N}$ a u mannia Bd. II., Heft $\mathbf{3}$, das Verzeichniss yon Hr. Gadamar, abgefasst werden, und wird so wenig Raum einnehmen.

\title{
Beobachtungen über die. Vögel des zoologischen Gartens zu Frankfurt a. M. Von
}

Lieut. Alex. von Homeyer.

Mit der systematischen Bezeichnung der Vögel ides : hiesigen seit sechszehn Monaten eröffneten zoologischen Gartens betrauet, habe ich oft Gelegenheit gehabt, denselben zu besuchen und Studien zu machen; leider. sind die:Notizen nicht so zahlreich geworden, wie ich es wünschte, woran vorzugsweise der letzte Sommer. Schuld war, der zum Beobachten, der Kriegsangelegenheiten halber, speciell für einen Offizier so ungünstig ,war. Wenn ich, daher die nachstehenden, anfänglich nur für meine Privatmappe bestimmten Aufzeichnungen der Oeffentlichkeit zu übergeben mich entschliesse, so geschieht dies nur nach wiederholten freundlichen Aufmunterungen und in der Voraussetzung, dass bei ihrer Beurtheilung ein nicht zn strenger Maassstab angelegt werde.

Der Vọllständigkeit halber führte ich sämmtliche Arten auf, welche sich im zoologischen Garten befinden, ${ }^{\text {in }}$ der Benennung leitete mich G. R. Gray's Systematik: Genera of Birds.

1. Gypaëtus barbatus (Storr ex L.). Der durch Dr. Alfred Brehm im Sommer. 185̆7 aus Spanien mitgebrachte junge Vogel starb im Winter desselben Jahres wahrscheinlich in Folge einer Erkältung. Ich glaube nämlich dies deshalb annehmen zu dürfen, weil er sich während des Sommers und Herbstes äusserst wohl befand, dann im Wịnter bei den ersten kalten Tagen zu kränkeln anfing, indem er die Federn. sträuble und nicht recht fressen wollte, und endlich bei der ersten schneidenden Kälte starb; was übrigens einen neuen Beweis zu der bekannten Erfahrung liefern: dürfte, dass. Kältegrade, welche von Thieren in: der Freiheit noch ganz gut ertragen werden, denselben Thieren in der Gefangenschaft, w\$o ihnen die entsprechende Bewegung feblt, bei einiger Dauer verderblich werden können. 
2. Neophron percnopterus (Sav. ex Li). Obwohl beide Aasgeier gleich alt, vielleicht sogar aus einem Nest sind, (Dr. A. Brehm brachte sie ebenfalls als junge Vögel aus Spanien,) so verfärbten sie sich doch zu sehr ungleicher Zeit. Der grössere begann damit im Frühling .59. Zuerst verfärbten sich die Schulterfedern, dann im Laufe des Sommers der Kopf, Kropf und Bauch in bräunlichweiss, and endlich im October die Flügeldecken wie die Nackenfedern in schmutzig Graugelb. - Der schwächere Vogel verfärbte erst im August die Schulter- und Bauchfedern, und hat jetzt im November noch Kopf, Kropf und Hals dunkel, fast schwarz. 'Ob die Verfärbung durch Mauser oder Umfärbung stattfand, konate ich nicht recht beobachten, doch lagen während der Sommerzeit stets Federn in der Volière. Die Füsse der jetzt $2^{1 / 2}$ Jahr alten Vögel sind röthlich. Sie sitzen auf der Erde fast wagerecht, auf dem Baume mit in der Regel gesträubten Halsfedern. Sie sind zahm aber heimtückisch. Herr Schöff von Heyden füttert sie oft mit Semmelkrumen, die sie begierig aus der Hand nehmen.

3. Sarcorhamphus Papa (Dum. ex L.). Siehe Journ. V. 51, hier ist der eigenthümlichen Zungenbildung des S. californianus Erwähnung gethan, die wir wenigstens ähnlich bei papa wiederfinden, nur vermisse ich die Mittheilung des rerdickten und gereifelten Obergaumens in der Weise, dass er eben dieselbe Form hat, als die Höhlung der halb cylindrisch-muldenförmigen Zunge, und beí geschlossenem Sehnabel vollkommen in diese hineinpasst. So erst scheint diese merkwürdige Doppel-Vorrichtung beim Fressen ganz besonders zum Abreissen von Fleischtheilen sich zu eignen.

4. Gyps fulvus (Gmel.). Die Zunge ist hier ungefähr dieselbe wie bei papa, vielleicht ist die Rundung flacher, der Obergaumen jedoch in der Weise verschieden, dass es nur ein etwas nach innen gerichteter harter Höcker (Zapfen) ist, welcher so vor der Zunge steht, dass er bei geschlossenem Schnabel die Höhlung derselben vorne an der rundlichen Spitze schliesst.

Höchst merkwürdige Stellungen nehmen diese Geier beim Sichsonnen ein. Mit weit ausgebreiteten, muldenförmig nach Innen gewendeten Flügeln kehren sie sich der Sonne zu, um so alle Strahlen aufzufangen, oft stundenlang sitzend, ohne den Körper zu bewegen, nur der Kopf dreht und wendet sich mit stolz um sich blickendem Auge. Bei der zweiten noch behaglichern Stellung sitzt der Vogel auf den Tarsen, die Flügel werden vorne an den Schultern 6 bis 10 Zoll gelüftet, während die Schwingen beim Sthwanze eng anliegen. Die Füsse sind weit vorgenommen, und hält der auf die Erde gestützte Schwanz 
das Gleichgewicht. Die dritte Stellung entsteht aus der zweiten dadurch, dass der Vogel sich hinlegt, dabei den Hals weit vorstreckt, sogar den Kopf auflegt und die Augen schliesst. Die Flügel liegen nicht fest an, und ist der Schwanz wenig fächerförmig ausgebreitet; mit dieser Stellung scheint mỉ gewöhnlich ein kleiner Schlummer verbunden zu sein.

Mit rothbraunen Milanen (Milvus regalis), die man zu ihnen der Gesellschaft halber in die geräumige Volière brachte, harmoniren sie vorzüglich.

5. Buteo vulgaris (Bechst. ex L.). Eine sehr schöne weisse Varietät (albidus) mit schwarzen Flecken und rostfarbenen Schulterfedern befindet sich mit grauen und dunklen Bussarden in einer Voliere. Ich beobachtete im Frühlinge einen merkwürdigen Kampf. Derselbe wurde auf der Erde ausgeführt, und batte die grösste Aehnlichkeit mit einem Hahnenkampfe, indem die Streitenden gegen einander sprangen, und sich mit den Fängen zu fassen resp. zu verletzen suchten.

6. Aquila fulva (Möhr. ex L.). Drei Vögel aus der Schweiz und Tyrol. Das von Schlegel „Vogels van Nederland" Seite 37 angegebene Kennzeichen, nach der Färbung des Schwanzes alt und jung zu unterscheiden (alt: Voorhelft mit grisachtige onregelmatige dwarsbanden als gewaterd und jung: Voorhelft wit) ist leicht und, soviel ich hier sah, richtig.

7. Aq. naevia (Möhr. ex L.) aus Pommern. Die Stimme des Schrei- und Steinadlers ist sich sehr ähnlich, sie klingt laut und voll: hspik - hspik bei letzterem fast hspiak, bei naevia ist der Ton nicht so rund, ein wenig höher und in der Folge des Tempo's schneller. Beim Schreien (naevia schreiet viel auf der Erde, fulva-mehr auf dem Baum) wird der Kopf nicht oder unbedeutend bewegt, der Schnabel ziemlich weit geöffnet und der Ton aus der Tiefe des Leibes hervorgestossen. Beide Adler baden gern, naevia sass sogar zu wiederholten Malen im Januar, Februar und März in seinem fast einen Fuss tiefen Badenapf, und badete sich entenartig, sogar den Kopf untertauchend.

8. Aq. Bonellii (Temm.). Als Nestrogel durch Alfr. Brehm aus Spanien. Ein herrlicher Yogel von dauerhaftem Naturell, an dem ich meine Freude habe; von allen Adlern vorzugsweise seines klugen königlichen Auges, wie auch seiner Zutraulichkeit wegen, mein Liebling. Das Jugendkleid im ersten Sommer ist dunkel rothbraun, und entbehrt fast jeglicher Zeichnung; im nächsten Sommer ist die Färbung die des jungen Vogels im Sulsemil'schen Werk Taf. 19, nur ist sie nicht ganz so fahl; im dritten Sommer die des alten Vogels (Taf. 18) im genannten Werke, nur stärker schieferblan überflugen, namentlich der Schwanz. 
9. Circaëtus gallicus (Gmel.), (siehe Naumannia VI. 202). Die Schlangenadler sind ruhige Vögel, die in aufrechter Haltung mit gelockertem Gefieder viel auf einer Stelle sitzen, ihr eulenartiges Auge ist jedoch stets lebhaft. Bei der Fütterung geberdeten sie sich sehr eigenthümlich, auf die hingeworfenen Fleischstücke stürzten sie sich eilig mit einem grossen Sprung, legten sich mit ausgebreiteten Flügeln darauf, und schrien laut und wohlklingend: plie - plie ${ }^{*}$ ), sich dabei viertelstundenlang ansehend. Fingen sie dann endlich $z u$ fressen an, so wurde bei jeder nur irgend fraglichen Bewegung eines Individuums das Manövre des Fleischdeckens voller Misstrauen wiederholt. Erst nach monatelangem Bekanntsein wuchs das Zutrauen, ohne jedoch die Vorsicht ganz ausser Acht zu lassen. (Dies eigenthümliche Treiben, wenn auch nicht sa ausgebildet, sah ich bei einigen Eulen: otus, aluco, noctua, ubbrigens erinnert es auch an den jungen Gyps fulous des Pfarrer Baldamus; siehe Naumannia 1. 1. 34.).

Im Sommer $d$. J. halte man 5 Schlangenadler:

1) Ein altes Weibchen mit zwei Jungen (aus zwei Nestern) aus der Bayrischen Pfalz (s. Journ. VII. 52) und

2) Zwei junge Vögel aus Nord-Afrika.

Die beiden jungen Pfälzer Vögel waren unter sich gleich gefärbt, unterschieden sich aber von den ebenfalls unter sich gleichen Afrikanern auf das Auffallendste ${ }^{* *}$ ), wie folgt:

$$
\text { aus der Pfalz: aus Nord-Afrika: }
$$

\section{Grösse:}

stärker.

schwächer.

2. Die dunkeln Theile

des Kropfes, Kopfes und Nackens:

graubraun.

fahl, gelblich, rothbraun.

3. Bauch:

rein weiss. gelblich weiss.

4. Flecken des Unterleibes:

nur wenige, klein und scharf braun. zahlreich, gross und fahl röthlich braun.

5. Rücken:

frisch braun. fahlbraun.

oder im Allgemeinen: der Farbenton der Pfälzer war graubraun und

*) Welcher Schrei mit dem des Buteo vulgaris zu vergleichen ist, er ist jedoch lauter, klar und rein, und nicht so näselnd.

**) Diese Verschiedenheiten fielen auf den ersten Blick in die Augen. 
kräftig im Colorit *), der der Afrikaner gelblich rothbraun und matt verloschen (wie verschossen). Die Zeichnung war bei allen Vögeln im Allgemeinen gleich. - Ich konnte nicht umhin, auf die interessanten Abweichungen der Färbung aufmerksam zu machen, und das Präsidium zu bitten, die Thiere unter jeder Bedingung für den zoologischen Garten zu behalten, da sie unter diesen Verhältnissen nicht als unnöthige Doubletten, sondern vielmehr als äusserst interessante klimatische oder lokale Verschiedenheiten **) zu betrachten wären, doch kam man leider meinem Wunsch nicht nach, und verkaufte die Afrikaner nach Dresden an den sich daselbst bildenden zoologischen Garten. So wurde mir die fernere Beobachtung unmöglich gemacht, anch starben die pfälzer Vögel im. Laufe des Summers; der eine brach beim Abreissen eines Bissens von einem grossen Fleischstück sich den Unterschnabel ab, die anderen starben wohl in Folge der unpassenden Fleischnahrung.

Die Färbung der Iris ist bei den Jungen ein lebhaftes Hellgelb, bei den Alten ein lebhaftes Hochgelb, daher nur nach dem Alter, nicht nach dem Klima abweichend. - Naumann spricht (Naumannia I. S. 5) von der Färbung der Iris vergleichsweise zu Strix bubo, ohne dieses höchst in die Augen springenden Unterschiedes zu erwähnen.

10. Haliaëtus albicilla (Sav. ex L.). Der Unterschied der Grösse zwischen Männchen und Weibchen ist aufallend; oft schreit das riesige Weibchen **** ) mit weit vorgestrecktem Kopfe laut: kak, kak, kak, und den Kopf zurückwerfend mit weit geöfnetem Schnabel leise: xsi - xsi - xsi, den Kopf wieder vorworfend sehr laut: kak kakkakkakkak - kak und nach einer kleinen Pause in ruhiger Haltung leise : kak.

11. Helotarsus ecaudatus (Smith ex Shaw). Zwei junge Vögel von diesem Jalire aus Afrika variiren in der Hauptfärbung. Der grössere, den ich $g$ nennen will, wahrscheinlich das Weibchen ist dunkler und brauner, als der kleinere $(k)$, welcher mehr grau ist. Diese Verschiedenheiten sind in diesem Herbst sehr deutlich am Kopf, Rücken und Flugel sichtbar, weniger am Unterleibe, hier sind jedoch die Tropfflecke, die beide Vögel nur am Unterleibe und Gurgel haben, bei $g$ weit bleiner und dunkler, ebenso ist die Kehle, die Stirn und die Augenbogen dunkel, fast schwarz, während diese Theile bei $k$ licht hell sind.

*) Die Färbung des alten Vogels war grauer und die Zejchnung markirter.

**) Individuell ist die Verschiedenheit wohl nicht, weil die Vögel je unter sich gleich.

***) Vom Männchen hörte ich nie einen To:. 
Die bei beiden Vögeln gleich gefärbte Wachshaut ist im September dunkelgrünspanfarbig, nach dem Schnabel zu gelblichgrün, bekommt aber im October bei dem grössern Vogel lebhaftrothe Flecke, die sich von unten durchzuarbeiten scheinen, indem sie anfänglich matt, dann deutlicher hervortreten. Die nackte Haut, die sich vom Schnabel um die Augen zieht ist bei beiden Vögeln gleich, aber lichter als die Wachshaut gefärbt, erscheint jedoch durch die vielen sie bekleidenden blau schwärzlichen Haare bläulich graugrün. Die Iris ist kastanienbraun, das Augenlid gelblichgrün, gleich dem äussern Rande der Wachshaut. Die Fürbung des Schnabels ist bis zur Krümmung gleich der Wachshaut, die Krümmung wie der Haken sind schwarz. Die Füsse sind im October merkwürdig verschieden gefärbt, bei $g$ fleischfarben, (namentlich die Tarse, das Grünliche kaum durchschimmernd), bei $k$ dunkel grünspanfarbig. Jetzt im November fangen die Füsse von $k$ auch an, röthlich zu werden, wie sich auch auf der Wachshaut einzelne feine rothe Fleckichen zeigen, welche jetzt bei $g$ sehr gross und deutlich sind. Hieraus geht hervor, dass die Umfärbung der Füsse und der Wachshaut von Grün in Ziegelroth in gegenseiligen Beziehungen stebt. Die Zunge steht in ihrer Bildung zwischen Adler- und Geierzunge mitteninne, der Höcker des Obergaumens, den wir bei den Adlern nicht finden, ist hier ganz in der Weise wie bei Gyps fulvus vorhanden.

Was das Naturell der Vögel anbelangt, so sind sie ganz harmlos und zahm, und lassen sie sich gern in den langen Kropf- und Halsfedern nach Ungeziefer suchen; in der Haltung gleichen sie einiger Maassen dem Circaëtus gallicus *). Nicht oft lassen sie einen Ton hören, dieser ist sanft, voll und nicht sehr laut: kwa, kwa, (die Stellung des Kopfes wird hierbei nicht verändert und der Schnabel wenig geöffnet). - In der Aufregung jedoch wirft der Vogel den Kopf hintenüber, sträubt die langen Halsfedern "schleiertaubenartig“ über den glatten Kopf, wodurch er ein äusserst merkwürdiges Aussehen erhält, und schreit leise, den Schnabel weit öfnend: kăk, und daun sehr laut: kăk - kaōh. Die hohen Töne hi, hi, hi, ki welche Alfred Brehm (Naum. VI. 200, wie auch Journal VI. 90) angiebt, hörte ich erst vor einigen Tagen zum ersten Mal, nämlich ein bussardartiges hiak - hiack - hiack, der Ton war leise, voll und nicht kreischend, und schien ein Freudenton zu sein.

12. Tinnunculus alaudarius (Vieill. Briss.). Mit Kalbfleich sind

*) Beim Sitzen auf der Stange stehen die äussern Zehen rechtwinkelig zur Mittelzehe, die innern im Winkel von 30 Grad zu dieser. 
diese lieblichen Falken auf die Dauer nicht zu erhalten, man muss zuweilen kleine. Vögel und Mäuse füttern.

13. Milvus regalis (Cuv. ex L.).

14. Milous niger (Cuv. und Briss). Der Schrei der jungen Vögel beider Milane ist wohl nicht zu unterscheiden, oder ist der von niger ein wenig rauher und kürzer?

15. Astur palumbarius (Lacep. ex L.).

16. Athene noctua (Boje-Retz), Die Scops Lempiji (Bp.), Strix Lempiji (Horsf.) muss mit unserer noctua, wenngleich im System nicht so nahe stehend, nach Dr. Bernstein's Mittheilung (Journ. VII. 181) unendliche Aehnlichkeit in ihrem Wesen haben, vorzugsweise in der Art und Weise des Rauben's.

17. Bubo maximus (Sibb.). Viele unserer Uhu's starben ganz plötzlich, ohne vorher Symptome des Unwohlseins merken zu lassen; hieran ist unpassende Nahrung Schuld, indem man ihnen nur Fleisch, höchst selten Vögel oder. Thiere mit Federn und Haaren giebt, was zur Verdauung nothwendig sein dürfte.

15. Syrnium aluco (ex L.). Wir besitzen stridula (L.), rufa (Gmel.) und Zwischenstufen, doch keine genau in der Mitte stehende.

19. Otis vulgaris (Flem. ex Cuv.).

20. Strix flammea (L.). Obgleich während des Tages fast immer schlafend, bemerkt sie doch den sich ihrem Käfig Nahenden.

21. Dacelo gigas (Leach-Bodd.).

22. Upupa epops (L.).

23. Sitta europaea (L.).

24. Paroides biarmicus (Koch ex L.). Ausserordentlich gewandte Vögelchen.

25. Turdus cyaneus (L.). S. Journ. f. Orn., VII. S. 317.

26. Anpelis garrulus (L.).

27. Gymnorhina leuconota (Gould!. Die verwandschaftlichen Beziehungen zwischen dem weissrückigen Flötenvogel und dem ächten tibicen (Lath.) liegen unendlich nahe, da beide Vögel meines Wissens nach in allen Beziehungen sich gleich, und nur durch die verschiedene Färbung des Rückens, die bei diesem weiss, bei tibicen schwarz ist, von einander $\mathrm{zu}$ unterscheiden sind, wie dies auch aus den vorzüglichen Abbildungen Gould's: Birds of Australia zu ersehen ist; ebenso scheint der Unterschied zwischen Männchen und Weibchen höchst unbedeutend zu sein. - Mir wurde das Vergnügen zu Theil eine junge einjährige G. leuconota zu sehen, indem der hiesige zoologische Garten eine solche durch Zufall in Hamburg acquirirte. Dieselbe zeigte bei sonst 
gleicher Zeichnung des alten Vogels, (nur waren die Ränder nicht so scharf,) die Farben nicht so rein, das Dunkle war nicht rein schwarz und das Helle, was sich besonders auf dem Rücken recht deutlich zeigte, nicht weiss, sondern hell-bläulich schieferfarben; im zweiten Jahre war diese Färbung sehr licht, fast weiss, mit einzelnen schieferfarbenen Kanten, und im dritten Jahre endlich rein weiss, wie auch die übrigen Theile glänzend schwarz. So glich er vollkommen dem alten Vogel des Professors Kaup, (s. Journ. f. Orn., IV. Jahrg., S. 460,) welcher ehenfalls für den zoologischen Garten acquirirt wurde. Die Zeit der Mauser scheint in den September zu fallen.

Wenn nun leuconota auch nicht der ächte Flötenvogel, so ist er dessen ungeachtet im sonoren Flöten vieler, oft sehr verschiedener pirolsartiger Strophen ein Meister, der Ton ist angenehm, voll und wird nie langweilig, man kann ihn übrigens durch tiefes Pfeifen mit dem Munde täuschend nachmachen, ich wenigstens unterhalte mich oft so mit meinem lieben Vogel, der mich zuweilen aus dem entlegensten Theil des Gartens zu sich za rufen sucht; komme ich, so legt er sich im Käfige auf den Rücken und wartet, bis ich ihn liebkose, dabei schliesst er dann wohlgefällig die Augen. Geh' ich fort, so lăuft er krähenartig schrittweise im Käfig auf und ab und springt auf eine Trittstange, um von Neuem mit Pfeifen zu beginnen.

Der Kaup'sche Vogel ist durchaus nicht so liebenswürdig, sondern hackt auf das Empfindlichste nach der Hand; sein Flötenton ist ein wenig tiefer.

28. Cyanocorax cristatus (Boie ex L.). Zwei Junge aus NordAmerika. Im August wechselten sie das Jugendgefieder des ganzen Körpers mit Ausnahme des Kopfes, mit diesem begannen sie erst im September so plötzlich, dass alle blauen Federkielchen stoppelartig zu gleicher Zeit zum Vorsthein kamen. Sie fressen gern allerlei Beeren; im Sommer schrieen sie oft ${ }_{n}$ schärp !"

29. Gracula religiosa (L.).

30. Pastor roseus (Temm. ex L.). Mauserte October und November. 31. Sturnus vulgaris (L.). Singt täuschend wie ein Canarienvogel.

32. Xanthornus aurantius.

33. Agelaius phoeniceus (Vieill. ex L.). Als Stubenvogel würde er durch seinen entfernt pirolsartigen Pfiff lästig werden, so aber nicht in einem zoologischen Garten, wo die kräftige Strophe sich herrlich ausnimmt, zwischen dem wirren Gezwitscher vieler kleinen Exoten und dem schreienden Gesange neuholländischer Papageien: kuku, ku, $\bar{i}$, ae, ist der einfache Pfiff, bei dem der Vogel sich hoch aufrichtet; die 
Federn des Rückens sträubt und den Schwanz etwas anziehend fächerförmig ausbreitet, dann werden einige Sprünge gemacht und der Pfiff beginnt von Neuem. Abends kurz vor dem Dunkelwerden hört man häufig ein lärmendes staarartiges ,spett, spett.“ tember.

34. Dolichonyx oryzivorus (Swains, ex L.), Verfärbung im Sep-

35. Hyphantornis larvata (Rüpp.).

36. Ploceus oryx (Cuv. ex L.).

37. Pl. franciscanus (1sert.). Beide Arten gedeihen ohne Sonnenschein nicht gut, wenigstens ist dieser während der Mauserzeit unentbehrlich; viel dauerhafter hingegen ist $\mathrm{Pl}$. melanogaster mit seinem Brunellen locktonartigem Gesange.

38. Pl. sanguinirostris (L.). Kräftige Burschen, die in der $\mathrm{V}_{0}-$ lière, ohne zänkisch zu sein, dominiren.

39. Vidua serena (Cuv. ex L.).

40. V. paradisea (Cuv. ex L.).

41. Cardinalis virginianus (Bp. ex L.).

42. Estrelda astrild (Swains. ex L.).

43. E. amandava (ex $\cdot$ L.).

44. E. benghala (ex L.).

45. E. minima (Vieill.).

46. Amadina fasciata (Swains. ex Gmel.).

47. A. oryzivora (ex L.).

48. A. nitens (ex L.).

49. A. Lathami (A. Smith.).

ว0. A. malacca (ex L.).

51. A. punctularia (ex L.).

52. A. maja (ex L.).

53. A. cucullata (Swains.).

54. A. sanguinolenta (Temm.).

55. A. cantans (Gmel.).

5́6. Spiza cyanea (Bp. ex L.). Der Indigo-Vogel hat einen fröhlichen und vollen Schlag, der zwischen dem Gesange unseres Troglodytes parvulus und Accentor modularis mitten inne steht.

57. Spiza ciris (Bp. ex L.). Hat man anderswo auch die Bemerkung gemacht, dass die Männchen viel zärtlicher sind, als die Weibchen?

58. Paroaria cucullata (Bp.). Eine fast schwarze Varietät, bei der auch das Rothe dunkler, als beim normal gefärbten Vogel war, starb beim Beginn des Winters. 
59. Gubernatrix cristatella (Less. Vieill.). Der Gesang ist laut und wohlklingend: spia, speut, spia, spia.

60. Nymphicus novae hollandiae (Wagl. ex Gmel.).

61. Platycercus eximius (Vig. Shaw.).

62. Pl. zonarius (Vig. Shaw.). Ein schöner harmloser Vogel.

63. Platycercus haematonotus (Gould). Hat einen ganz vorziiglichen Gesang, welcher in vieler Hinsicht an Tardus musicus erinnert, wenngleich dies ubertrieben, da der Ton nicht sehr laut und kreischend ist, so lassen sich doch Beziehungen nicht verkennen.

64. Pl. melanurus (Wagl.). Der vorige Vogel singt, dieser pfeift ganz vorzüglich mannichfaltig menschenartig, und zwar so täuschend, dass ich oft mit Vergnügen sah, wie. Leute hierdurch vollkommen getäuscht wurden.

65. $P l$. Barnardii (Vig. Lath.).

66. Pl. scapulatus (Vig. Bechst.). Ein junges Männchen mit noch grünem Kopfe begann im Laufe dieses Herbstes den Kopf in Roth umzufärben, eine Mauser fand nicht Statt. Die Umfärbung hat mich sehr interessirt, einzelne Federn waren nämlich an der Wurzel roth und an der Spitze grün, andere in umgekehrter Weise, andere zeigten die eine Seite grün, die andere roth, während noch andere nur rothe Flecken hatten; als Vermittlerin beider Farben trat gelb auf. Hierdurch erhielt der Kúpf (im November) ein eigenthümlich scheckiges Aussehen.

67. Palaeornis Alexandri (Vig. ex L.).

68. Pal. torquatus (Briss.).

69. Pal. pondicerianus (Temm. Pl. enl.).

70. Melopsittacus undulatus (Gould ex Shaw.). Der zoologische Garten erzielte nur ein Junges, glücklicher war mein verehrter Freund, der Advocat Dr. Binding, welcher in den letzten Jahren 17 Junge (merkwürdiger Weise 14 Männchen und 3 Weibchen) züchtete. Die hierüber gemachten Beobachtungen wurden meinem ebenfalls hochverehrten Freunde, dem Dr. Carl Bolle, zur Verfügung gestellt, welcher dieselben mit seinen eigenen, und den Beobachtungen Anderer verschmolzen, in dem Juli-Hefte dieses Journales unter dem Titel: „Beginnende Domestication des Undulatus-Papageien", veröffentlicht hat.

71. Euphema elegans (Gould). Singt auch recht angenehm, doch nicht so schön und anhaltend, wie haematonotus.

72. Ara ararauna (Briss. ex L.).

73. A. aracanga (Gmel.).

74. A. macao (L.).

75. A. severa (L.). 
76. A. Illigeri (Kuhl.).

77. Conurus carolinensis (Kuhl ex L.).

78. C. aurifrons (Less.).

79. Eclectus Linnaei (Wagl.).

80. Psittacus erithacus (L.).

81. Chrysotis aestivus (Swains. ex L.).

82. Chr. amazonicus (Gmel.).

83. Chr. pulverulenta (Gmel.).

84. Psittacula passerina (Briss. ex L.).

85. Ps. roseicollis (Vieill.).

86. Cacatua rosea (Briss-Vieill).

87. C. moluccensis (Gmel.). Wir besitzen 4 Vögel, alle zeichnen sich durch ihre ausserordentliche Zahmheit und Gutmüthigkeit aus.

88. Cacatua cristata (L.).

89. C. sulphurea (Grmel.).

90. Cuculus canorus (L.).

91. Columba oenas (L.).

92. C. palumbus (L.). Beide Arten wurden so zahm, dass sie gegen das Gitter ihrer Volière fliegen, um den Kindern die Brotkrumen aus der Hand $z u$ nehmen.

93. Columba leucocephala (L.).

94. C. domestica (L.). Vorzüglich schöne Perrücken-, Eis-, Struppund Hühnertauben etc.

95. Geopelia malaccensis (Swains. ex Gmel.). Sonnen sich gern in sehr artigen Stellungen, indem sie sich auf die eine Seite legen, den gegen die Sonne gerichteten Flügel ausbreiten, und vollkommen so über den Rücken zurückschlagen, dass die Sonnenstrahlen nicht allein die Seite bescheinen, sondern auch aufgefangen werden.

96. Oena capensis (Selby ex L.).

97. Turtur auritus (Selby ex Ray).

98. T. risorius $\left(\mathrm{L}_{\text {. }}\right.$.

99. T. aegyptiacus (Lath.).

100. Ocyphaps lophotes (Gould). Wunderschön in Gould's ${ }_{\text {Birds }}$ of Australia" abgebildet. Ihr Pfalzton ist ein volles: uk, uk.

101. Phaps chalcoptera (Selby ex Lath.).

102. Penelope superciliaris (lllig.).

103. P. marail (Lath.). Ich würde mich nicht unterfangen nach den Abbildungen von Spix's ,Aves Brasiliae" beide Arten zu bestimmen, weshalb ich mich nach den Exemplaren der senkenbergischnaturforschenden Gesellschaft richtete, welche nach Mittheilung des Con- 
servators Herrn Erkel por wenig Jahren durch Prinz Bonaparte geordnet resp. berichtigt wurden, wie auch nach den Exemplaren des Berliner Museums. Die Penelopiden sind recht ausgezeichnete Vögel: der lange Schritt, die wagrechte Haltung des Körpers, das wenige Neigen des Schwanzes, das stolze Tragen des Kopfes, den sie zuweilen eigenthümlich schütteln, lassen fasanenartige Beziehungen nicht verkennen. Das Gefieder ist mehr oder minder glänzend. Der Ton ist weich, klagend und gurgelnd in der Art und Weise wie bei Crax alector und Pauxi mitu, welche gurgelnden Töne vielleicht allen Cracidae eigen sind.

\section{Crax alector (L.) ${ }^{*}$ ).}

105. Pauxi mitu (L.).

106. Pavo cristatus (L.). Ein prächtig altes Männchen verlor sein Leben auf höchst merkwürdige Weise, es wurde nämlich, als es sich zufällig gegen Abend auf den Kletterbaum im Bärenzwinger setzte, um dasellsst zu übernachten, ron der geschickten Katharina, der Bärin, mit Umsicht gefangen und gefressen.

107. Meleagris gallopavo (L.).

108. Phasianus colchicus var. torquatus, ein Vogel, der in jeder Beziehung dem Edelfasan gleicht, jedoch einen weissen Ring hat, es ist dies wohl nur eine zufällige Varietät, ein interessantes Nachgebilde des ächten Phas, torquatus (Temm.).

109. Phasianus torquatus (Gmel.). Es unterliegt wohl keinem Zweifel, dass dieser durch den Ring, wie vor dem unächten Ringelfasan durch die eigenthümliche fahle Färbung ausgezeichnete Vogel, von seinen Verwandten (wenigstens den gewöhnlichern Arten) die schönste Form und die edelsten Bewegungen hat.

Das Weibchen legte wohl 50 , theilweise sehr verschieden gefärbte Eier. Wenn man viele Eier einer Species (ganz abgesehen vom Ringelfasan) vor Augen hat, so sieht man deutlich, dass obwohl alle einer und derselben Art angehören, dieselben doch oft unter sich von höchst verschiedener Grundfärbung sind. Die Natur hat hier, wie so oft die Grenzen nicht so nahe gezogen, sie hat eine solche Abweichung der Species erlaubt, ohne jedoch zu weit zu gehen. Bis jetzt nun glaubte ich, dass diese Verschiedenheit rein individuell sei, dass ein und derselbe Yogel immer ein (was die Grundfärbung anbetrift) gleichgefärbtes Ei lege, wenigstens bei ein und demselben Gelege, dass aber die Ver-

") Die schöne Crax Blumenbachï (Spix) wurde für einen sehr geringen Preis in Hamburg zum Kauf angebolen, wurde jedoch, weil man schon alector besass und der Ylatz mangelte, nicht acquirirt. 
schiedenheit der Gelege unter sich durch Alter, Temperament, Nahrung, Aufenthalt, Klima etc. bedingt sei. Erst neuerdings babe ich bemerkt, dass dies nicht immer der Fall ist, indem ein Weibchen des Phasianus torquatus, dessen Eifärbung die des colchicus mit seinen Abweichungen ist, sämmtliche Färbungen legte, die überhaupt nur bei der Species zulässig, nämlich am:

9. April ein gelbliches $\mathrm{Ei} ; 10$. April ein bräunlich grünes Ei; 11. April ein röthlich braunes Ei; 12. April ein olivfarbiges Ei; 15. April ein grünbraunes Ei; 16. April ein olivbraunes Ei.

Die Verhältnisse waren vor und während dieser Zeit dieselben, und ohne dass sie sich änderten, legte das Weibchen eine Zeit lang beständig ,olive" Eier, dann wieder alle oben genannten Abänderungen. Auf die Fruchtbarkeit hatte dies in keiner Weise Einfluss, denn grade aus den oben angeführten Eiern schlüpften fast ohne Ausnahme allerliebste junge Fasanen aus, welche sich vor den Jungen des colchicus wie des var. torquatus einerseits durch geringere Grösse, wie aber vorzugsweise auf den ersten Blick durch ihr warmes, gelbliches Colorit unterschieden. Diese gediehen während des Sommers ausgezeichnet, und erhielten durch die im Anfang des Herbstes stattindende Mauser ein den Eltern sehr ähnliches Kleid, so namentlich die Männchen den Ring.

110. Phasianus pictus (L.).

111. Ph. Nycthemerus (L.).

112. Gallus domesticus (L.). In reicher Auswahl, namentlich die seltenen Muschelhühner (grau mit.muschelförmig hufeisenartigem Kamm), die Riesen-Strupphühner, blaue und schwarze Polands mit weisser Haube, Jerusalemer Hühner etc.

113. Numida meleagris (L.).

114. Perdix cinerea (L.). Es macht keinen üblen Eindruck, wenn der alte Hahn mit braunem Schilde in der Carière durch das hohe Gras einer Wiese läuft, auf einem Hügel Halt macht, sein lautes „Kirrgick" ruft, und ebenso schnell wie gekommen wieder in der nächsten Strauchanlage verschwindet.

115. Coturnix communis (Bonn. ex $L_{2}$ ). Man sagt immer, dass zwei zusammengesetzte Männchen nicht schlagen, hier waren vielleicht 10 und ebenso viele Weibchen in einem grossen Käfige beisammen, und hörte man den fröhlichen Schlag "Flick die Büchs" immerfort.

116. Caccabis rufa (Kaup ex L.). Das Rothhuhn bäumt sehr gern und benimmt sich dabei durchaus nicht ungeschickt.

117. Ortyx virginiana (Steph. ex L.). 
118. Ortyx cubanensis (Gould). Die Färbung beider Arten ist nicht so brillant, als die der gehäubten $O$. californica, doch ist sie äusserst geschmackvoll vertheilt. Der Ruf von $O$. virgin. ist voll, laut, aber klagend, von der 0 . cuban. mir unbekannt. Die Zeichnung beider Vögel ist fast gleich, ebenso zeigt die Färbung viel Uebereinstimmung, cubanensis ist dunkler und am Vordertheil fast schwarz, dabei das helle daselbst perlförmig und rein weiss, während virg. hier braun ist, und das helle mehr gestrichelt und nicht rein weiss hat; - jedenfalls stehen sich die Arten unendlich nahe, so viel ich weiss, sind auch die Eier einander sehr ähnlich. (Siehe übrigens Journ. IV. 337).

119. Callipepla californica (Wagl. ex Lath.). Ein Weibchen legte im Laufe des Sommers (nur kurze Zeit zuweilen aussetzend) 75 (fünfundsiebenzig) Eier. Diese theilweise durch Zwerghühner, Gold- und Silber-Bantam's bebrïtet, waren fast alle fruchtbar. Die Eier sind wachteleierartig, die Grundfärbung ist lichter (ähnlich der Perdix saxatilis-Eier) und die Flecken röthlichbraun (wie wir sie bei den Waldhühner-Eiern finden), nur einige arteten in lila oder violet aus, dann schien mir aber die Schaale ein wenig rauher, ich möchte sagen staubiger (wie bei den Silberfasan-Eiern) zu sein. Die Form ist die der Wachteleier in der Regel „ein wenig mehr kreiselförmig ", in der Grösse dieselben etwas übertreffend. - Die kleinen zierlichen wachtelähulichen Jungen gediehen anfänglich gut, starben aber alle mit Beginn der kalten Nächte. Zusammengesetzte alte Männchen vertragen sich gut, und pfalzen sogar zusammen, wobei sie sich auf der Stange sitzend hoch in die Höhe richten, sich ein wenig bücken und volle, helle und weitklingende Töne hören lassen, die durchaus jeglichen melancholischen Charakters entbehren.

Alle 3 Collins gehen gern und viel auf die Bäume, das Aufbäumen geschieht sehr geschickt, oft sitzen sie stundenlang auf einer kaum fingerdicken Sprosse; auf der Erde laufen sie sehr zierlich und gewandt, obwohl etwas trippelnd, das Köpfchen dabei wenig neigend und hebend, was sich namentich bei der californica, des Federschmuckes halber, sehr schön ausnimmt, und dem Vogel ein gar zierliches Aussehen verleiht.

120. Struthio camelus (L.). Ein herrliches Riesen-Paar, welches gewöhnlich in Eintracht, zuweilen jedoch auch so in Zwietracht lebt, dass man es trennen muss. Nachdem im Winter 58/59 das Weibchen von den Wänden vielen Mörtel losschlug und verschluckte, legte es einige Eier, ebenso später im Sommer, nachdem die Begattung zu wiederholten Malen vollzogen, welche man jedoch nicht auszubrüten verstand. Ein welsches Huhn (Meleagris gallopavo) musste sogar Ver- 
suche machen, nachdem man das $\mathbf{E} \mathbf{i}$ halb in Moos verpackte, diese waren jedoch erfolglos, trotzdem man sich nicht die Mühe verdriessen liess, das Ei regelmässig zu wenden.

121. Rhea americana (Möhr. ex L.). Männchen und Weibchen. Beide, wahrscheinlich Geschwister wurden in Winter 57-58 im zoologischen Garten zu Antwerpen gezogen, und noch im selbigen Jahre dem hiesigen Garten überlassen; dieselben gedeihen ausserordentlich gut. Das Männchen macht zuweilen ganz eigenthümliche Sprungmanövres; bei heiterem Sonnenschein lief das Paar gern mit gelüfteten Flügeln oder es sonnte sich liegend mit angezogenen Beinen und senkrecht aufgerichtetem Halse.

122. Dromaius emu (Vieill. Steph.). Legte im Januar und Februar sechs ausgezeichnete schöne smaragdgrüne Eier. Bemerkenswerth ist vielleicht, dass der sonst in der Regel grüngrau gefärbte Koth, während der Zeil des Eierlegens einerseits selr flüssig wurde, andererseits aber auch dieselbe intensiv grüne Färbung wie die Eier zeigte; was sogar so weit ging, dass der Koth einen Tag vor und einen Tag nach dem Eierlegen am intensivesten gefärbt war. - Von den übrigens sehr normalen Eiern zeigte das erste die hellste Farbe, wie die schwächsten Poren und Körnung, das letzte hingegen, welches vom Gelege das kleinste, war sehr dunkel, und von ausgezeichnet schöner, körniger Struktur.

123. Casuarius galeatus (Vieill. ex L.). Es - ist ein Männchen, das Abschälen des Höckers hat mich interessirt, dies begann im Herbst 1858 damit, dass die vordere Bogenkante sich auftrennte, die beiden entstandenen Ränder nun lösten sich von vorne nach hinten zu nach und nach immer mehr ab, während sie aber auch vorne successive abgingen. Dieser Process ging so langsam von statten, dass im Januar kaum die Hälfte, im April dreiviertheil, und erst im August das Ganze geschehen war. - Dieses Schälen findet nicht alle Jahre statt, denn jetzt im November ist noch nicht wieder von neuem damit begonnen, während im vorigen Jahre schon im September damit der Anfang gemacht wurde.

124. Otis tarda (L.). Junge Vögel aus Thüringen gediehen während des Sommers bei Getreide, Mais und Brod, untermischt mit gehacktem Kohl yortreflich, mit Beginn des Herbstes machten sie jedoch bedenkliche Gesichter und sind jetzt todt.

125. Oedicnemus crepitans.

126. Vanellus cristatus.

127. Psophia crepitans (L.). Siehe die vorzüglichen Studien des Professors Burmeister (Journ. II. 67). Der erste Trompetenvogel, den ich sah, liess mich auf den ersten Blick die verwandschaftlichen Bezie- 
hungen speciell zu den Fulicarien wie auch zu Anthropoödes erkennen, nicht so seine Beziehungen zum Dicholophus sive Cariama, es mag sein, dass die anatomischen Verhältnisse der Art sind, was aber die äussere Erscheinung, die äussern Merkmale anbetrifft, so will mir die Vereinigung beider zur Gruppe Psophiinae nicht recht gefallen.

128. Cariama cristata (Briss. ex L.). Dieser in jeder Beziehung merkwürdige, von den Naturforschern in der Systematik so verschieden untergebrachte Vogel, der Beziehungen zu den Lauf-, den Sumpf- wie den Raubvögeln zeigt, erinnert auch in vieler Hinsicht speciell in seiner äussern Erscheinung an die amerikanischen Waldhühner, die Cracidae. Das Gefieder ist weich und locker anliegend, die verlängerten Halsfedern, welche oft geschüttelt und gesträubt werden, dass sie fast senkrecht abstehen, geben dem Vogel ein eigenthümliches Ansehen. In der Ruhe ist der Hals angezogen, der vordere Theil des Leibes gehoben, und der Schwanz geneigt; beim Laufen legt der Vogel sich sehr vorne in die Brust, hierdurch bekomml der Körper wie der zusammengelegte Schwanz eine wagrechte Haltung und erinnert so sehr an die Abbildung des Cursorius chalcopterus (Temm.) in Gray's: Genera of Birds. Der leichte, gewandte und schnelle Lauf lässt recht die grosse Schnellkraft der Beine wie die verwandschaftlichen Beziehungen zu Cursorius sehen, die Flügel liegen hierbei an, selbst beim schnellsten Lauf. - Kommt der Vogel in Hader, so macht er tolle Sprünge, er sträubt den Hals, bläht sich raubvogelartig auf, und breitet den scharf mit schwarz gezeichneten Schwanz, während eines Sprunges in der Luft, ihn seitwärts schwenkend, fächerförmig aus; auch wird hierbei, vielleicht um nicht das Gleichgewicht zu verlieren, zuweilen der eine oder der andere Flügel ausgebreitet, so wird bald springend, bald laufend der Gegner unter den drolligsten Manövern angegriffen und verfolgt, der Schnabel ist hierbei als die eigentliche Waffe zu betrachten, indem er durch einen glicklichen Griff oft viele Federn ausrupft, während der oft vorgeschnellte Fuss nie krallt, sondern nur Stösse, Fusstritte giebt. - Diese sich oft wiederholenden Zwistigkeiten sind nie von langer Dauer, auch nehmen sie nie einen bösartigen Charakter an, nur einmal fand eine wirkliche, wochenlange Feindschaft mit einem Silberfasan statt. - Sperlinge, junge Ratten und Mäuse, die sich dem Futterkasten nähern, werden oft, indem der Vogel im schnellsten Lauf auf sie stürzt, mit unendlicher Geschicklichkeit gefangen, und, nachdem sie erst im Wasser vollkommen eingeweicht und mundrecht gemacht worden sind, mit Haut und Haaren verschluckt. Dies Einweichen geschieht vorzugsweise immer bei grösseren Sachen, wie Ratten und Sperlingen, seltener bei 
den kleineren, den Mäusen. - Während des Tages bäumt der Cariama sehr selten auf, er steht, geht oder läuft, und giebt sich nur selten oder nie der Ruhe in der Art hin, wie es beispielsweise die Kraniche gern zur Mittagszeit thun; die Nacht hingegen bringen sie stets auf einem Baume, nie auf der Erde zu; gegen Abend bäumen sie ziemlich ungeschickt auf, denn oft verlieren sie das Gleichgewicht *) und springen wieder auf den Boden, sitzen sie aber einmal auf ihrem Platz, der immer derselbe ist und wohl nie gewechselt wird, so ziehen sie die Beine wie den Hals an, und verbringen in so gekauerter Lage die Nacht in aller Ruhe.

Das Geschrei ist ebenso merkwürdig wie unser Vogel selbst, es ist "raubvögelartig", ungemein kreischend und laut, und bei ruhigem Wetter eine halbe Stunde weit hörbar; hierbei sitzt der Vogel am liebsten etwas erböht, etwa auf einem 4-5 Fuss hohen Baumstumpf. Auf der Erde sitzend, ist das Geschrei weniger laut und anhaltend, auch wird der Kopf hierbei nicht bewegt; springt der Vogel aber auf einen jener auf der Wiese stehenden Baumstumpfe, so mögen sich alle Nervenschwache möglichst schnell entfernen, denn es beginnt jetzt im wahren Sinne des Wortes: ein Schrei-Concert. Es besteht aus zwei Theilen, beim ersten nimmt der Musiker eine aufrechte Haltung an, sieht gen Himmel, und schreiet mit sehr heller gellender Stimme unendlich laut: ha - hahahahi - hihihi - hiel - hiel - hi - el worauf eine kleine Pause von $3- \pm$ Secunden eintritl und mit einem kleinen Nachruf: hăk endet. Bei jeder der einzelnen Silben wird der Kopf wechselseitig angezogen und gehoben, wodurch eine eigenthümliche Bewegung des vorderen Theils entsteht, dann wird der Kopf vollkommen hintenuber geworfen, und der zweite Theil geschrien, dieser beginnt noch viel lauter und endet dann schwächer werdend, er klıngt ungefahr:

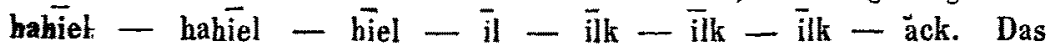
ăck ist wieder leise und ist ganz so, wie der Nachruf des ersten Theils. - Der Vogel schreiet oft $1 / 2$ Stunde lang, er beurkundet übrigens auch seinen Siun für Musik dadurch, dass er sich vorzugsweise hören lässt, wenn ein Concert im zoologischen Garten ist, indem er die Musik begleitet **).

*) Es ist möglich, dass diese Ungeschicklichkeit vielleicht vom Amputiren herrührt.

**) Man erzählt hier Hanches über seine geistigen Fähigkeiten, was in Vergleich zur Psophia crepitans sehr interessant wäre, vielleicht später Einiges darüber. 
129. Grus cinerea (Bechst. ex L.).

130. Anthropoïdes virgo (Vieill. ex L.).

131. Balearica pavonina (Briss. ex L.). In allen Bewegungen ist der Kronenkranich nicht so graziös wie virgo, aber viel lebhafter, muthwilliger und fröhlicher, $A$. virgo ist die seltsame Jungfrau, $B$. pavonina der ausgelassene Jüngling, er tanzt und springt (oft mit ausgebreiteten Flügeln) unter den merkwürdigsten Bnwegungen und Stellungen, macht Sprünge von 3 bis 4 Fuss Höhe und ist stets guter Dinge, weshalb ich ihn fast der stillen $A$. virgo vorziehe.

Im Juni und Juli d. J. rief er oft in aufrechter Haltung, ohne den Kopf zu bewegen und den Schnabel zu öffnen; mit voller, lanter und sehr wohlklingender Stimme: rāg - räg — räg (wohl 20 Mal), welchen Ton man mit kräftiger Tenorstimme leicht nachahmen kann. Während der Herbstmonate hörte ich ihn nur selten, der Angstlaut, den er zum Beispiel ausstösst, wenn man ihn greift, ist sehr laut aber unangenehm kreischend: argargargarg im schnellen Tempo. - Die Stimme der A. virgo ist ganz anders und durch Silben unendlich schwer wiederzugeben, der laute Ton ist scharf und jubilirend und etwa durch tirrr oder terrr wiederzugeben. - Als Merkwürdigkeit ist noch anzuführen, dass die B.pavonina im März mit einem Mal die Neigung zeigten, auf einen Fuss stehend in dem fast $3 / 4$ Fuss tiefen, mit Wasser gefüllten Bassin, zu übernachten, was der sehr kalten Witterung halber natürlich sehr auffiel.

132. Ardea cinerea (L.).

133. A. purpurea (L.). Von der Knoblauchs-Aue bei Darmstadt (Journ. VII. 57). Während des Tages ist der Purpurreiher nicht so munter wie $A$. cinerea, er steht gern "rohrdommelartig" geduckt stundenlang neben einem Strauch, ohne sich zu bewegen, welchen Platz er auch nur verlässt, wenn man ihn stört, dann eilt er zuweilen sich umsehend im langen Schritt davon. Mit einbrechender Dunkelheit wird er lebhafter, indem er behende um den Weiher schleicht.

134. Ardea minuta (L.). Mir wurden zwei Fälle bekannt, dass sich der Zwergreiher, wahrscheinlich glaubend, dass er nicht gesehen wurde, mit den Händen ergreifen liess. Auf diese Weise gelangte vor mehreren Jahren ein einjähriges, vollkommen gesundes Weibchen, welches auf der Trebel, einem Fluss Pommerns nahe am Ufer auf dem Blatt einer Nymphaea alba sass und zu schlummern schien, in den Besitz meines Bruders Ernst. Dieser durch mich oft erzühlte und zuweilen auch wohl bezweifelte Fall wiederholte sich in diesem Jahre im hiesigen zoologischen Garten, indem ein Wärter in der Nähe des Bärenzwingers ein zweijähriges Männchen, das über den Weg schlich und sich 
in einen Strauch duckte *), mit den Händen ergriff. Dieses eben so muntere wie komische. Vögelchen befindet sich noch jetzt im wohlen Zustande hierselbst.

135. Botaurus stellaris (Steph. ex L.). Die Rohrdommeln nehmen oft eine eigenthümliche pfahlartige Stellung ein, indem der Hals wie auch der Schnabel schräge nach oben gerichtet in der Verlängerung des Leibes stehen. Die lebhaften grünlichen Augen, die sehr tief nach unten sizzen und daher nach vorne sehen können, beobachten aus dieser Stellung eine jegliche meiner Bewegungen, wobei der Hals, der wechselseitig aufgeblasen und schlank gemacht wird, kaum sichtbar gedreht wird. Die Füsse sind hierbei $x$-förmig eingeknickt. Die Rohrdommeln erinnern in ihrer äussern Gestalt, ihren Bewegungen wie in allen ihren Eigenthümlichkeiten sehr an die Rallen, und dürfte sie diess diesen. Vögeln ebenso nahe bringen, wie sie die Verschiedenartigkeit des Ei's entfernt.

136. Nycticorax griseus (Steph. ex L.). Die beiden Nachtreiher sitzen gern stundenlang ganz nahe an einander geduckt auf einem Baumstumpf, zuweilen schreien sie unter merkwürdigen Geberden mit rauher Stimme: rhāka - rōka - rak. — rhäk - rak.

137. Platalea leucorodia (L.). Sie wissen mit ihrem breiten Schnabel die Regenwürmer, die dicht unter dem Rasen einer Wiese sitzen, sehr geschickt hervorzuholen. Der Schnabel des jungen, scheinbar schon ausgewachsenen Vogels ist nur vielleicht 3-4 Zoll lang, und erhält derselbe erst im Laufe des Spätsommers die Länge der Alten (Naumannia II. 2. 59). Dann auch erst bekommt der Vogel den Ton des alten, während er.bis dahin den Kopf verneigend, immerfort oft zum Ueberdruss : pierrr, pierrr schreiet.

138. Ciconia alba (Briss. ex L.).

139. Ciconia nigra (Bechst. ex L.). Die Kropffedern der jungen Vögel sind noch im zweiten. Jahre grau, während das übrige Gefieder schon schön metallisch glänzt:

140. Numenius arquatus (L.) ***).

141. N. phaeopus. (L.). Ein lieber munterer Vogel, welcher

*) Der Zwerg-Rohrdommel duckt sich stets im Strauche selbst, indem er hineinschlüpft.

**) Die beiden Numenius-Arten sind schüne und sehr dauerhafte Vögel, welche sich auf einer Wiese sehr gut ausnehmen, limosa, Tolanus, Tringa etc. sind hingegen sehr' zärtlich und' wohl kaum für die Dauer zu erhalten, eine Ausnahme hiervon macht bei uns ein Weibehen des Machetes pugnax, welches seit 2 Jahren wohlauf und munter ist.

Joura. f Ornith., Vit. Jahrg, Nix. 41, September 1859. 
nicht so ernst wie arquatus ist, und seinen fröhlichen, weitschallenden Ruf : dike - - dike in schnellem Tempo hören lässt.

142. Limosa rufa (Briss.).

143. Totanus calidris. (Bechst. ex L.). Lockte im Sommer oft Abends beim Dunkelwerden : djü̈b - djü klingend, aber etwas klagend.

144.: Tringa canutus (L.).

145. Machetes pugnax (Cuv. ex L.).

146. Gallinula chloropus (Briss. ex L.).

147. Fulica atra (L.).

148. Phoenicopterus antiquorum.(L.). Thr Geschrei ist bald gänse-, bald kranichartig (später vielleicht ausführlicher).

149. Chenalopex aegyptiacus (Steph. ex L.). Sie sind nicht soviel auf dem Wasser wie anf dem Lande; nur ein Mal (am 16. October d. J.), sah ich, wie alle vier Gänse mit der grössten Leichtigkeit und Gewandheit tauchten, dabei blieben sie lange unter dem Wasser und schienen sich spielend zu verfolgen, dessen ungeachtet muss diess doch als eine Ausnahme angesehen werden. Die Stimme ist dumpf und klingt: hōh oder. hāh, sie ist oft so heiser; dass es nur ein rauhes Hauchen ist, indem man den eigentlichen Ton nicht einmal hört. Kurz. vor der Paarung benimmt sich das Männchen höchst entenartig, indem es immerfort 'mit dem Kopf nickt und datdatdatdatdat sagt.

150. Anser segetum (Gmel.). Eine schöne Gans, welche viel auf dem Wasser ist und sich durch ein ernstes, ruhiges aber nicht träges Betragen characterisirt. Die 4 Exemplare variiren nur unbedeutend am Schnabel, indem sich das Gelb etwas verschieden. vertheilt.

151. Anser albifrons (Gmel.). In der Brustzeichnung variiren sie ungemein, zuweilen ist sogar die Färbung so ungleich vertheilt, dass die eine Seite schwarz, die correspondirende grau gefärbt ist. Wir besitzen auch eine weissstirnige Gans mit rosarothen Füssen, welches wohl nur roseipes (Schleg.), sive pallipes (De Selys, Naumannia V. 264) sein kann, sie unterscheidet sich .jedoch nach den angegebenen Kennzeichen einerseits durch ihre Kleinheit, indem sie fast 3 Zoll kleiner als die zwei weiblichen albifrons ist, andererseits durch ihren Schrei, indem dieser von dem der albifrons durchaus nicht abweicht.

153. Anser cygnoides (L.).

154. Bernicla Brenta (Steph. ex Pall.). Aeusserst zahme und liebenswürdige Thierchen, welche Gras aus der Hand nehmen oder mit weit vorgestrecktem Kopf und geöffnetem Schnabel leise zischen.

155. Bernicla leucopsis (Bechst.). Viel phlegmatischer. 
146. Bernicla inornata (King.). Es sind Vögel von diesem Jahre und in London gezüchtet. Der Schnabel ist schwarz, der Kopf und oberer Theil des Halses grau, die Stirn lichtgrau, das Auge dunkel, der untere Theil des Halses, die Brust und der entsprechende Rückentheil röthlich graubraun : mit schwarzen Wellen. Der Bauch ist weisslichgrau mit dicken schwarzen Querwellen, die Mitte des Bauches von der Brust bis zum After reinweiss, Rücken steinbraun mit einzelnen grossen rothbraunen und schwarzen. Wellen (fasanartig geschuppt); die Schulter ist weiss, Spiegel dunkelgrün, unten weiss gesäumt, röthlich schillernd; Schwingen schwarz; Schwanz schwarz, Unterschwanzdeckfedern röthlich kastanienbraun; Tarse äusserlich ziegelroth, innerlich wie die Schwimmhaut und Nägel schwarz. - Ich gab diese Beschreibung des Vergleiches halber mit dem in Gray's Gen. of Birds abgebildeten, alten Vogel, bei dem die Färbung brillanter und die Zeichnung markirter ist.

156. Bernicla canadensis (Steph. ex L.). Vier prächtige Vögel schwimmen äusserst friedfertig zwischen all’ dem kleinen Entengeflügel, von Zeit zu Zeit ihren lauten Schrei: aāa $-a \bar{a} a, a \vec{a}-\bar{a}-\mathbf{a} \bar{a} a$ hören lassend, welcher jedoch selten in seiner Vollständigkeit, in der Regel nur theilweise gegeben wird. Da dieser gewöhnlich von allen Vieren wiederholt wird, so artet er leicht in einen Lärm aus, und muss derselbe in Amerika, wo während der Zugzeit oft Tausende beisammen sind, furchtbar werden können. Auf dem Lande stehen sie oft auf einem Fusse mit weit zurückgelegtem Hals und vorgestreckter Brust und haben so, in ihrer Haltung viel Aehnlichkeit mit dem Schwan.

Sämmtliche Gänse (vielleicht $A$. aegyptiacus ausgenommen) sind äusserst friedfertige Thiere, die unter sich wie mit Jedermann, nur nicht mit den Herrn Botanikern in Harmonie leben, weil sie gern die Bäume und Sträucher, namentlich die jüngern, auf das Schonungsloseste benagen und schälen, ohne nach der. Etikette des seltenen Gewächses zu sehen; vor Allen geht hier die B. canadensis mit bösem Beispiel voran, so hat es wenigstens einer ihrer Hauptfeinde, welcher mit besonderer Liebe die Anpllanzungen überwacht, beobachtet.

158. Cygnus atratus (Lath. ex L.). Das Tauchen nach auf dem Grunde des Weihers wachsenden Pflanzen ist anders wie bei $C$. olor, er stellt sich nämlich wirklich auf den Kopf, den Bürzel ,entenartig" in die Höhe gerichtet, was ich beim weissen Schwan nie gesehen zu haben glaube.

159. Tadorna vulpanser (Leach. ex L.). Dr. Bodinus versicherte mich, dass die Brandente wie auch andere zärtlichen Enten nicht anders auf die Dauer zu erhalten seien, als mit einem täglichen $Z_{u s a t z}$ von 
kleingeschnittenem rohen Fleisch. Ihre Stimme im etwas eiligen Tempo klingt eigenthümlich dumpf: kokkokkokkok, und in der Aufregung cur - cur - curr. Hat man viele Brandenten anf einem Weiher, so sieht man sie nie zusammenschwimmen; sondern fast immer uber den ganzen Weiher einzeln vertheilt, was sich ihrer schönen markirten Färbung halber zwischen den andern Enten immer sehr vortheilhaft ausnimmt. Mit.dem Tauchen hält sie es ähnlich wie die ägyptische Entengans.

160. Aix sponsa (Boje ex L.).

161. A. galericulata (Boje ex L.). Als etwas Originelles verdient wohl erwähnt zu werden, dass sich, als die weibliche $A$. sponsa starb, die weibliche A. galericulata sofort an das Sponsa-Männchen anschloss, und jegliche Annäherung oder Zudringlichkeit ihres eigenen Männchens beissend abwies. Man hoffte, interessante Bastarde zu erzielen, doch kam es nicht einmal zum Eierlegen, woran wohl das Amputirtsein Schuld ist, was die Vögel verhinderte, in der Höhe in einem Nistkasten zu brüten, und sie zwang unten auf der Erde zu nisten, was gegen ihre Natur ist. Es ist demnach rathsam, beim Ankauf nicht amputirte Vögel auszuwählen *).

162. Mareca penelope (Steph. ex L.). Die Erpel lassen oft den bekannten pfeifenden Ton: ui hören, der eigenthümlich lispelnd schnarrend endet und dann virr klingt. Der Hals ist dabei senkrecht aufgerichtet, der Schnabel weit geöffnet, und der Ton kräftig herausgestossen, ohne den Kopf zu bewegen. In der Aufregung pfeift der Erpel in schnellem Tempo nicht sehr laut: $\bar{u} \bar{i}-\bar{u}-\bar{u}-\bar{u}$.

163. Dafila acuta (Leach ex L.). Die Paare halten dicht zusammen; zuweilen lässt das Männchen seinen wohlklingenden Lockton: frü oder färü hören (oft im Frühlinge, sonst selten), wobei es den Kopf hochhebt, den Hals in eine schöne längliche S-Form bringt, ond nun mit dem Schnabel einen kurzen Ruck nach unten macht, wozu der Schwanz den Takt schlägt. 'Zur Begattungszeit hebt sich das Männchen neben. diesen Geberden zuweilen eigenthümlich hoch aus dem Wasser heraus, dass nur noch der hiutere Theil des Leibes im Wasser ist; ausserdem hörte ich ganz eigenthümliche, leise näselnde Töne, die mir vom Weibchen herzurühren schienen.

164. Anas boschas (L.).

165. Querquedula crecca (Steph. ex L.).

*) In Holland soll man aus gewinnsüchtiger Absicht die Weibchen der Mandarinente, welche ins Ausland verkauft werden, häufig kastriren und somit zur Fortpfianzung untüchtig machen. 
166. Querquedula circia (L.).

167. Chaulelasmus sireperus (Gray ex L.). Gar schön nimmt sich das schieferfarbene Männchen aus, es ist edel in der Gestalt wie in seinen Bewegungen; höchst characteristisch ist neben dem weissen Spiegel die markirte Schnabelzeichnung des Weibchens. Das Paar hält treu zusammen. Im Frühling lässt das Männchen mit hochaufgerichtetem Kopf ein volles, wohlklingendes pak hören, dem das rauhe $r$ (park) und das Schreiende unserer Anas boschas fehlt.

168. Spatula clypeata (Boje ex L.). Die Löffelenten, welche im Laufen Schnabel und Leib sehr wagerecht, den Hals angezogen halten, sind: durchaus nicht ungeschickt in ihren Bewegungen auf dem Lande.

169. Cairina moschata (Flemm. ex L.).

170. Fuligula cristata (Steph. ex L.).

171. Nyroca ferina (Flemm. ex L.). Beide Tauchenten liegen viel auf der. Mitte des Weihers, ohne viel umherzuschwimmen, nur zuweilen gehen sie vorzugsweise, um sich zu somen ans Land. Beim Tauchen springen sie förmlich aus dem Wasser heraus, um mit dem Kopf voran dann fast eine Minute lang zu verschwinden. Nur ein Mal hörte ich von der weiblichen $N$. ferina einen Ton, vom Männchen nie, (ich habe viele Stunden vergebens darnach gelauscht); dieser klang rirrrrr - a; - Bei dem rirrr warf sie den Kopf zurück, bei a wieder vor.

172. Clangula glaucion (Flemm. ex L.). Eine frisch eingefangene Ente (Weibchen) benahm sich erstaunlich wild und starb, trotz aller angewandten Mühe, sie mit Fischrogen zu stopfen, wohl in Folge von Angst. Sie tauchte nämlich so lange, bis sie endlich so ermattet war, dass sie auf dem Wasser liegen blieb und sich mit den Händen aufnehmen liess, unfähig, weder zu schwimmen, noch zu tauchen.

173. Anas domestica. In vielen Verschiedenheiten und Rassen wie Stein-, Smaragd-, Pinguin-, Riesen-, Zwerg-, Doppel- und krummschnäblige Enten. Ich liebe diese zahmen Vögel nicht im zoologischen Garten, indem sie mir zwischen das wilde Entengeflügel nicht recht zu passen scheinen.

174. Larus marinus (L.).

175. Larus argentatus (L.). Beim Schreien haben beide Möven dieselben Geberden, indem sie den vorgestreckten, aufgeblähten Hals bis zur Erde neigen, und während dieser Bewegung (nebelkrähenartig) den Ton hervorstossen, welcher bei $L$. marinus sehr tief: $\bar{o} g$, bei letzterer etwas höher, kreischender: ōg klingt. Die Mantel-Möve stösst dieses ög zuweilen 10-12 Mal sehr hastig hinter einander aus, was fast wie ein entferntes Hundegebell klingt; L. argentatus thut diess 
nicht, dafür hat sie aber andere, kreischende Töne, welche ich bei L. marinus nie hörte.

Oefters sah ich, wie $L$. argentatus -einen beim oder im Fressnapf sitzenden Sperling fing, denselben durch einen Schnabeldruck tödtete und sofort verschlang, ohne auch nur eine Feder übrig zu lassen.

176. Larus canus (L.). Hielt sich aicht.

177. L. ridibundus (L.)

178. Graculus carbo (L.). Neun Vögel durchwühlen den Grund des Weihers. Ich glaube irgendwo von Dr. Alfred Brehm gelesen zu haben, dass im Vergleich zum Schlangenvogel unser Cormoran im Tauchen und Schwimmen schwerfällig genannt werden kann, ich muss gestehen, dass ich da gern einen Schlangenvogel sehen möchte, denn mich überrascht der Cormoran noch tagtäglich durch seine Tauchfertigkeit, und doch sehe ich ihm schon fast 2 Jahre zu.

Im Weiher sind dreipfündige Karpfen, welche, obgleich als Nahrung zu gross, doch immerfort tauchend verfolgt werden. Ein Cormoran tauchte einem nach, erfasste ihn bei der Rückenflosse, hob ihn zur Hälfte aus dem Wasser, und liess ihn der Schwere halber wieder fallen. Der Karpfen kaum frei, suchte möglichst schnell zu entwischen, doch tauchte der Cormoran, nachdem er kaum zu Athem gekommen, wieder in die Tiefe, und das Spiel begann von neuem, bis der Vogel vollkommen ermüdet war, und der Fisch halb todt an die Oberfläche kam, um Luft zu schöpfen.

Das Schwimmen der Scharbe geschieht sehr verschieden, zuweilen hebt der Vogel den Schwanz hoch aus dem Wasser, wobei der Körper hoch oben auf der Oberfläche liegt, während andrerseits der Körper so tief liegt, dass das Wasser zuweilen über den Rücken hinweggeht, und der im Wasser selbst liegende, halbfächerförmig ausgebreitete Schwanz als Ruder dient; stets wird aber der Hals senkrecht und der Schnabel so gehialten, dass die Spitze (der Haken) ein wenig höher, als der Kopf selbst ist.

Das Tauchen geschieht vom Schwimmen selbst aus, oder auf die bei Anas ferina beschriebene Weise, hier ist der Sprung jedoch noch höher, und daher das Ganze noch augenscheinlicher.

Vom vielen Tauchen wird der Cormoran vollständig nass, er eilt, wenn er genug hat, möglichst schnell dem Lande, einem Stein oder irgend einem erhabenen Platze zu, und breitet der Sonne zugewendet die Flügel aus, dieselben sanft fächelnd, um sich zu trocknen. (Ich hörte, Dr. Jul. Hoffmann hätte hierüber neulich etwas im Buch der Welt veröffentlicht). Nach einer Viertelstunde ist das Werk geschehen, und 
sie beginnen mit dem Sichsonnen in derselben Stellung, jedoch ohne die Fligel zu bewegen.

Im Frühlinge trugen die Cormorane viel Reisig, zusammen, um ein Nest zu bauen, auch versuchten sie, junge am Weiher wachsende Strüucher abzubrechen; dessen ungeachtet bam es nieht ein. Mal zum Eierlegen. Sitzen mehrere Vögel beim Neste zusammen, oder tragen zwei Vögel einen Strauch gemeinschaftlich, (denn nur in diesen Fällen beobachtete ich es), so. stossen sie unter komischen Geberden tiefe, froschartig quackende Töne aus, die so recht zur eigenthümlichen Belebung eines Sumpf-Concertes beitragen mögen, namentlich wenn .Rohrsänger, , Rohrdommeln und Frösche mit einfallen.

Gegen die andern Vögel des Weihers zeigen sie sich in jeder Beziehung verträglich, dessen ungeachtet sind jene doch wenn diese tauchen, ängstlich, und suchen so schnell wie möglich ans Land zu kommen, hiervon sogar nicht ausgenommen ist der: schwarze Schwan, welcher ohne Aufenthalt durch schnelles Schwimmen: in einen andern Theil des Weihers zu gelangen sucht.

179. Pelecanus onocrotalus (L.). Der Pelikan schwimmt, trotzdem er im System als Nachbar steht, ganz anders als die Cormorane, er liegt nie so tief im Wasser, sondern hoch oben auf mit gelüfteten Fligeln „schwanenartig“.

Frankfurt a. M., im Herbst 1859.

Seltame Tonhuhinheit einer Ban-t-Eule, Strix barbata Pall:, Str. lapponica Sparrm. - Der bier gemeinte Fall gehört, unter die Zahl derjenigen, in Betreff deren es sich der Mühe lohnen würde, dass einmal Jemand die aufallendsten sammelte, ihre wahrscheinlichsten Ursachen zu ermitteln suchte und für diese Zusammenstellung den Titel wählte: die:gelegentlichen Thorheiten mancher Vögel. Denn offenbar ist die Veranlassung, sie zu begehen, den Umständen gemäss verschieden. Sie haben aber das mit einander gemein, worin eben das Wesentlichste der Sache liegt. Nämlich sie bezeichnen stets eine bedeutende, weun auch nur zeitweise Störung der Se elenthätigkeit des Thieres, die es theils für den Augenblick, theils für längere oder kürzere Zeit als nach seiner Weise "unzurechnungsfähig" erscheinen lässt. Diess giebt ihnen daher ein gar nicht unbedeutendes Interesse für die Thierseelenkunde.

Am bekanntesten sind jene wahren „Tollheiten, ${ }^{\circ}$ die manche A u erhähne während ihrer Begattungszeil nicht bloss dann und wann, son- 